\title{
Design parameters study on the stability and perception of riding comfort of the electrical motorcycles under rider leaning
}

\author{
Hsien-Chung Lai ${ }^{\text {a }}$, Jing-Sin Liu ${ }^{\text {a,* }}$, D.T. Lee ${ }^{a}$, \\ Li-Sheng Wang ${ }^{b}$ \\ a Institute of Information Science 20, Academia Sinica, Nankang, Taipei 115, Taiwan, ROC \\ ${ }^{\mathrm{b}}$ Institute of Applied Mechanics, National Taiwan University, Taipei 106, Taiwan, ROC
}

Received 1 October 2000; accepted 9 August 2001

\begin{abstract}
Owing to noticeable differences of the characters between the electrical and the conventional gasoline-powered motorcycles, such as heavier in weight, lower in mass center position and slower in forward speed, the control and design considerations of the electrical motorcycles are not the same as conventional motorcycles. In this paper, the rider upper body lean control system is considered as the only control action to analyze and simulate the stability and the perception of riding comfort of the rider-motorcycle system in different riding conditions and parameter designs. The equations of lateral motion of an electrical motorcycle, which includes the rider upper body leaning motion, are derived first from Newton mechanics. Via this dynamics model and its associated transfer functions at various speeds, perception of riding comfort is defined as the damping ratio of frequency domain Bode plots in classical control theory. By the analysis, we find that the tendency of the stability is the same under the straight-line and the cornering motions, but shows significant differences for different parameter designs of the electrical motorcycle. We discuss the effect on the perception of riding comfort issue for changing mass center position, total weight of the motorcycle, the wheelbase, the front fork rake angle and the front wheel trail distance of the motorcycle. On the premise of the stability, we use the maximum damping ratio to represent the riding response of the motorcycle to find the best perception of riding comfort. By the analysis results, if we move the mass center forward, shorten the wheelbase, steepen the front fork rake angle and shorten the front wheel trail distance of the current design of electrical motorcycles in the market, a better perception of riding comfort is achieved. From the user's point of view, our analysis
\end{abstract}

\footnotetext{
${ }^{*}$ Corresponding author.

E-mail address: liu@iis.sinica.edu.tw (J.-S. Liu).
} 
method of perception of riding comfort can be a supplement to the analysis of the stability of motorcycles.

(C) 2002 Published by Elsevier Science Ltd.

Keywords: Electrical motorcycles; Rider-motorcycle system; Stability; Comfort

\section{Introduction}

The studies of the dynamics of the vehicles and rider-motorcycle system in particular have long been of academic and industrial interest [4,5,7]. Some of the useful results are from the work of Sharp [1], Weir [2] and Mears [3]. In 1971, Sharp created the equations of motion of the two-rigid body motorcycle model by the Lagrange equation, and discussed the effect of the stability caused by the variations of the design parameters. Sharp brought up three main modes to analyze the motions of the motorcycle:

1. wobble mode of high frequency vibration motion;

2. weave mode of low frequency vibration motion;

3. capsize mode of nonvibration motion.

Sharp used the changes of the damping of the three modes to determine the stability of the motorcycle. In 1978, Weir and Zellner discussed the relationship of the control parameters of the rider and the motorcycle on the lateral-directional dynamics. They used the Newton Mechanics to derive the equations of motion of the motorcycle. In 1988, Mears added the rider upper body lean model to the motorcycle model of Weir, and discussed the effect of the moment of inertia of the wheels on the stability. In the discussion of Mears, we can know that the rider can stabilize the motorcycle by using only the rider upper lean motion. These works present a consistent phenomenon that the stability of the motorcycle will become unstable at low speed, stable even under 'hands free' control (control without applying steer torque) at moderate speed, and oscillatory unstable at high speed. The past researches were devoted to the stability analysis of the motorcycle, the effect of the change of the design parameters and the rider control simulation of the conventional motorcycle. On the other hand, the discussions on the perception of riding comfort issue and the analysis of the electrical motorcycles are lacking and are the focus of this study.

One character of the electrical motorcycles different from the conventional ones is that electric motorcycles are heavy vehicles with low payloads. The weight of the electrical motorcycle is centralized in the bottom of the chassis where the lead-acid batteries and the DC brushless motor are placed. Due to this, the mass center of the electrical motorcycles is lower than those of the conventional ones, which have upper oil tank and engine placements [7]. In addition, the usage of four lead-acid batteries as power source makes the mass of the electrical motorcycle also heavier than the conventional $125 \mathrm{cc}$ gasoline-powered motorcycles widely used in Taiwan. Furthermore, the maximum forward velocity of the electrical motorcycles is usually 
below $60 \mathrm{~km} / \mathrm{h}$, and the frequently operating speeds lie between 25 and $55 \mathrm{~km} / \mathrm{h}$, which lie in the range of mediate speeds of $125 \mathrm{cc}$ motorcycles. These factors cause the design consideration of electrical motorcycles to be different from the conventional motorcycles.

In view of the rapidly developing market for the electric vehicles due to increasing environment protection concerns all over the world and the possibility of regenerative braking and zero energy use at idle, the research on electrical motorcycles or scooters is important and needed. The researches on the aspects of the stability and comfort under various rider control behaviors for straight running $[3,6,8,9,11]$ or steady turning [13-15] two-wheeled vehicles have reached some solid results for further study of electrical motorcycles. For straight running motorcycles, Sharp [1] has arrived at the conclusion that they can be controlled with "hands off" at medium and high speeds. Cossalter et al. [13] investigated the effects of design (geometric and inertia) parameters and tire properties on steering torque in steady turning [14]. In the interest of comfort, this study considers the effect of using only the upper body leaning motion (moving left or right) to achieve stability and perception of riding comfort of electrical motorcycles in constant-speed forward and/ or constant radius cornering motion for various design parameters. We use the frequency domain Bode plots of the rider-motorcycle system transfer function as the basic methodology to analyze the relative stability and perception of riding comfort issues of rider-motorcycle system. Here, perception of riding comfort is defined as the damping ratio of the rider-motorcycle transfer function and is used as a performance evaluation criterion of the design parameters of electrical motorcycles. In particular, we investigate the effect on the perception of riding comfort for changing mass center position, total weight of the motorcycle, the wheelbase, the front fork rake angle and the front wheel trail distance of the motorcycle. Some design guidelines of electrical motorcycles are obtained by our analyses of perception of riding comfort.

This paper is organized as follows. In the following section a description of dynamic model for the rider-motorcycle system and a preliminary discussion about the control action is presented. In Section 3, the stability and perception of riding comfort of the rider upper body leaning control under straight-line and cornering motions are discussed. In Section 4, the effects of main design parameters of motorcycles on perception of riding comfort are analyzed quantitatively via Bode plots. Section 5 is devoted to the conclusions, and the major parameters and the notations used in this paper are listed in Appendix A.

\section{The ridermotorcycle system}

\subsection{Motorcycle model}

Observing the electrical motorcycles sold in the market and with the analysis method of motorcycles of the work [1-3], and including the rider upper 
body lean motion, we divide the rider-motorcycle system into three main parts as follows:

1. Front fork assembly: front fork, handle bar, front wheel, shock absorber.

2. Rear assembly: rear wheel, motor, batteries, chassis, the lower body of the rider.

3. Rider upper body: the upper body of the rider including hands and head.

Because the equations of motion involve many variables, it is hard to analyze. Due to this, we have some basic assumptions to simplify the rider-motorcycle model:

1. We divide the rider and the motorcycle as three rigid bodies as described above. The front fork assembly and the rear assembly are connected by the steering column, and the rider upper body and the rear assembly are connected by the fore and aft axes of the rider's waist. The rotating inertia of wheels and motor are taken into account.

2. We assume that the motorcycle is moving forward in a level ground with a constant velocity. Because of this, the pitch and bounce motions of the motorcycle are ignored.

3. The drag force of the motorcycle caused by the air is related to the direction and the velocity of the motorcycle. Because the velocity of the electrical motorcycle is slow, the effect of drag force caused by air is much less than the tire force. Therefore, the effect of the drag force caused by the air is neglected.

\subsection{The coordinate system of the electrical motorcycle}

Because the three parts of the rider-motorcycle system have relative motion, three coordinates are defined separately, one for each part (Figs. 1 and 2). The three coordinates can be transferred to find the relationship via the transfer matrix.

\subsubsection{Coordinate system}

Three coordinate systems are defined:

1. $x, y, z$ : Rear assembly coordinate system. The rear assembly coordinate system is a body-fixed coordinate system with the origin fixed directly on the ground level below the mass center of the rear assembly with the $x$-axis forward, $y$-axis rightward and the $z$-axis downward.

2. $x^{\prime}, y^{\prime}, z^{\prime}$ : Front fork assembly coordinate system. The front fork assembly coordinate system is a body-fixed coordinate system with the origin fixed on the front wheel axis, obtained by front fork rake angle $\lambda$ relative to $x y$-plane, followed by a positive steer angle rotation about the $z^{\prime}$-axis.

3. $x_{\mathrm{r}}, y_{\mathrm{r}}, z_{\mathrm{r}}$ : Rider upper body coordinate system. The rider body coordinate system is a body-fixed coordinate system with the origin fixed directly below the mass center of rider upper body on the height of the seat, and all the axes are originally parallel to the rear assembly coordinate, followed by a positive lean angle rotation about the $x_{\mathrm{r}}$-axis. 


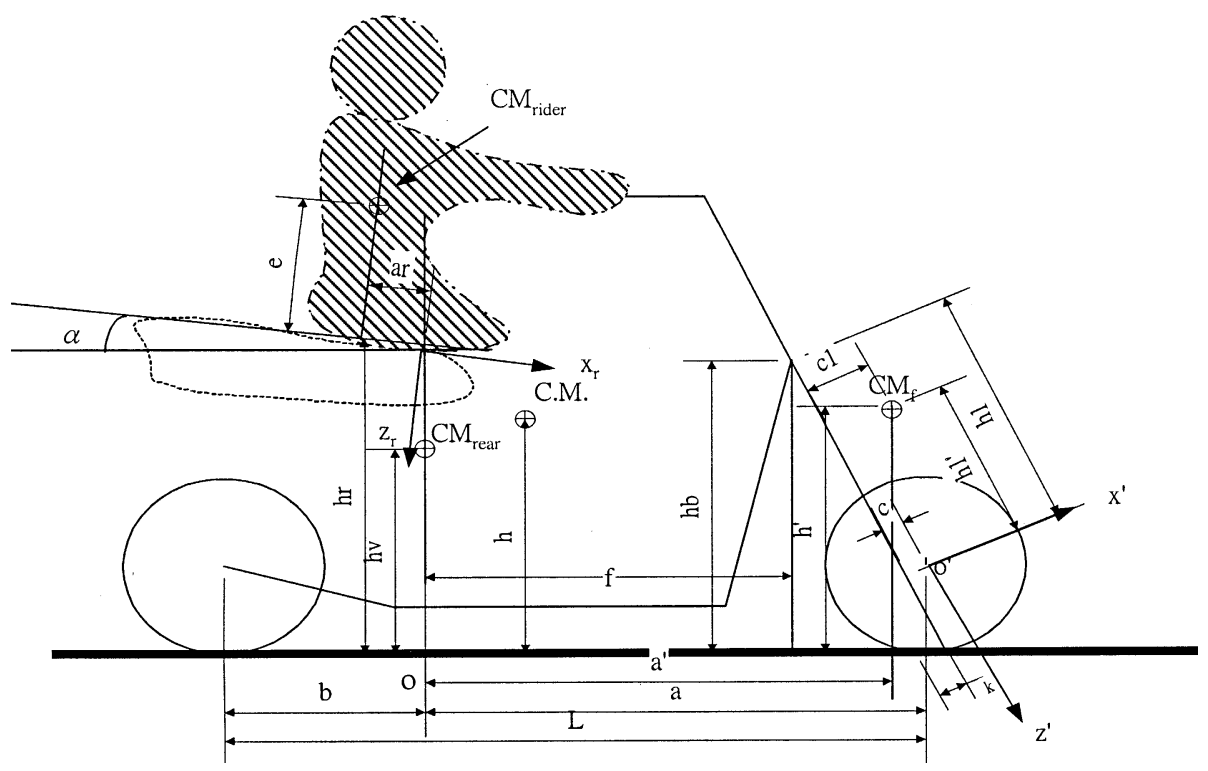

Fig. 1. The dimensions of the rider-motorcycle system.

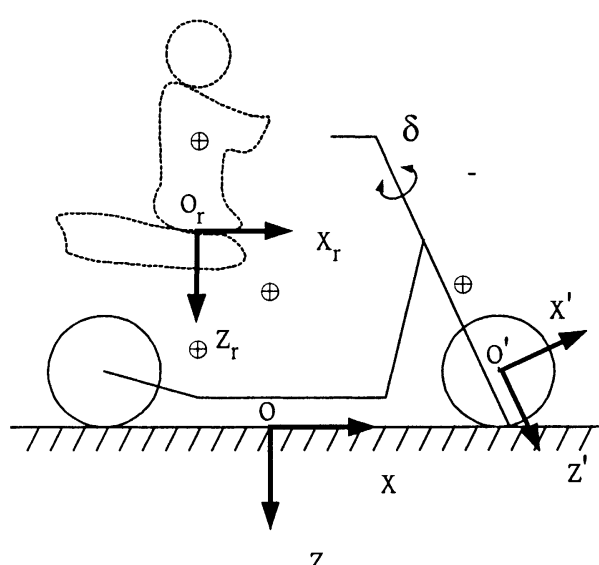

Z

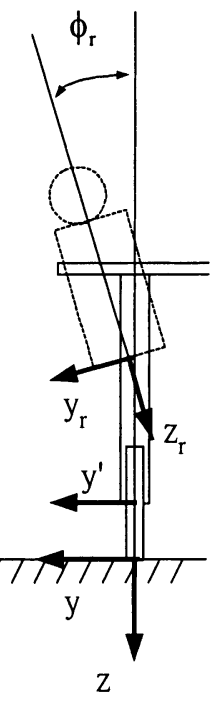

Fig. 2. The coordinates of the rider-motorcycle system.

\subsubsection{Coordinate transformation}

Mathematically, the coordinate transformation from the front fork assembly coordinate to the rear assembly coordinate is given by: 


$$
\begin{aligned}
& {\left[A_{\lambda}\right]=\left[\begin{array}{ccc}
\cos \lambda & 0 & -\sin \lambda \\
0 & 1 & 0 \\
\sin \lambda & 0 & \cos \lambda
\end{array}\right],} \\
& {\left[A_{\delta}\right]=\left[\begin{array}{ccc}
\cos \delta & \sin \delta & 0 \\
-\sin \delta & \cos \delta & 0 \\
0 & 0 & 1
\end{array}\right],} \\
& \omega_{x y z}=\left[A_{\lambda}\right]^{\mathrm{T}}\left[A_{\delta}\right]^{\mathrm{T}} \omega_{x^{\prime} y^{\prime} z^{\prime}},
\end{aligned}
$$

where $\omega_{x y z}, \omega_{x^{\prime} y^{\prime} z^{\prime}}$ are the angular velocities of the rear assembly with respect to the fixed coordinate system and body fixed coordinate system, respectively. $A$ denotes the rotation matrix.

In a similar manner, the transformation from the rider upper body coordinate to the rear assembly coordinate is given by

$$
\begin{aligned}
& {\left[B_{\phi_{\mathrm{r}}}\right]=\left[\begin{array}{ccc}
1 & 0 & 0 \\
0 & \cos \phi_{\mathrm{r}} & \sin \phi_{\mathrm{r}} \\
0 & -\sin \phi_{\mathrm{r}} & \cos \phi_{\mathrm{r}}
\end{array}\right],} \\
& \omega_{x y z}=\left[B_{\phi_{\mathrm{r}}}\right]^{\mathrm{T}} \omega_{x_{\mathrm{r}} y_{\mathrm{r}} z_{\mathrm{r}}} .
\end{aligned}
$$

Fig. 3 provides a schematic drawing of the rider-motorcycle system. The components of the motorcycle include the control elements and the rider-motorcycle system. Its dynamics is characterized by ordinary differential equations with control inputs. The dynamical equations of the motorcycle essentially follow Weir and Zellner [2] and Mears [3], which is a modification of Sharp's theoretical model [1]. The mass and inertial contributions of the rider are included in the free control equations and the steer torque and the rider lean control effects arise in the control element. It is described in terms of a 4 d.o.f. system, i.e., the lateral velocity $y$, the roll

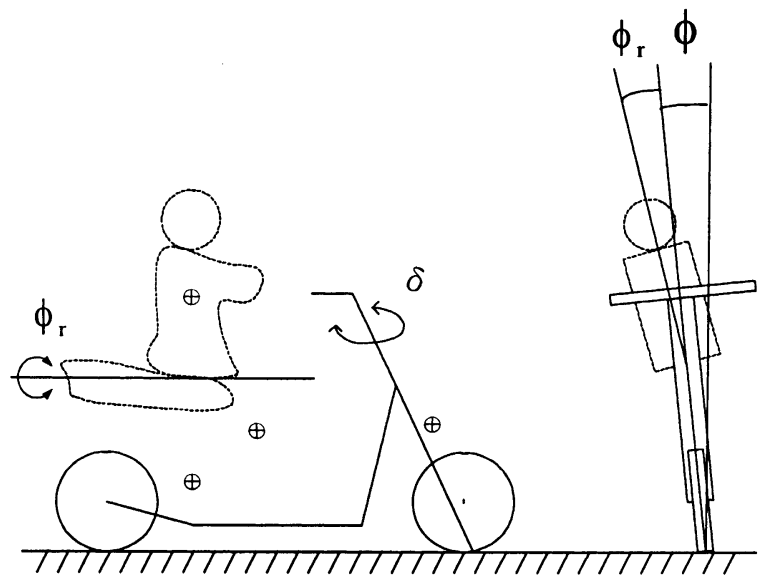

Fig. 3. The top front and side views of the rider-motorcycle system. 
angle $\phi$, the yaw rate $r$ and the front fork steer angle $\delta$. The variables of these equations are $y, \phi, r, \delta$, as described above and the front and rear tire forces $Y_{1}$ and $Y_{2}$. The front and rear tire forces are functions of the slip and camber angles, and have a lag effect decided by the relaxation length of the tire. The dynamics of the rider is modeled by 1 d.o.f., with the rider upper body lean angle being $\phi_{\mathrm{r}}$. In these equations, the influences of the front and rear suspension dynamics and aerodynamics are assumed to be negligible.

\subsection{Equations of motion}

The equations of motion for motorcycle directional dynamics with tire forces included as derived by Weir, Mears and Sharp are linearized equations of motion, invoking the assumption of small slip and camber angles when the motorcycle is under straight-line-running motion. This involves considering small perturbations about an initial operation point, deleting products of perturbation variables, and letting $\sin \theta \fallingdotseq \theta$ and $\cos \theta \fallingdotseq 1$. It is noticed that a real motion of a rider-motorcycle system on the city road is a combination of a series of straight-line and cornering motions. The slip and camber angles may not be assumed small and some of the products of perturbation terms may affect the lateral directional equations because of the cornering motion. This requires re-derivation of the equations of motion by Newton's law as Weir and Mears did but without any linearization.

\subsubsection{Derivation of equations of motion}

Using Newton's law and Euler's equation, and with the coordinate transformation and the kinematics' relationship, we can derive the equations of motion of the rear assembly, front fork assembly and the rider upper body with respect to moving coordinate systems, separately, as below:

Equation of motion for rear assembly:

$$
\begin{aligned}
& F_{\mathrm{v}}=m_{\mathrm{v}}\left(\dot{v}_{0}+\omega \times v_{0}\right), \\
& N_{0}=\dot{L}_{0}+\omega \times L_{0}+\dot{L}_{\mathrm{G}}+\omega \times L_{\mathrm{G}},
\end{aligned}
$$

where $F_{\mathrm{v}}$ and $N_{0}$ are all the forces and moments acting on the rear assembly. $m_{\mathrm{v}}$ is the mass of the rear assembly. $v_{0}$ is the velocity of the mass center. $\omega$ is the angular velocity of the rear assembly. $L_{0}$ and $L_{\mathrm{G}}$ are the angular momenta of the mass center of the rear assembly and the rotating parts (i.e., motor, wheel), respectively.

Equation of motion for front fork assembly:

$$
\begin{aligned}
& F^{\prime}=m^{\prime}\left(\dot{v}_{0}^{\prime}+\omega \times v_{0}^{\prime}\right), \\
& N_{0}^{\prime}=\dot{L}_{0}^{\prime}+\omega^{\prime} \times L_{0}^{\prime}+\dot{L}_{\mathrm{G}}^{\prime}+\omega^{\prime} \times L_{\mathrm{G}}^{\prime},
\end{aligned}
$$

where $F^{\prime}, N^{\prime}$ are all the forces and moments acting on the front fork assembly; $m^{\prime}$ is the mass of the front fork assembly; $v^{\prime}$ and $\omega^{\prime}$ are the velocity and the angular velocity of the front fork assembly; $L_{0}^{\prime}$ and $L_{\mathrm{G}}^{\prime}$ are the angular momenta of mass center of the front fork assembly and the rotating parts, respectively. 
Equation of motion for rider upper body:

$$
\begin{aligned}
& F_{\mathrm{r}}=m_{\mathrm{v}}\left(\dot{v}_{0 \mathrm{r}}+\omega \times v_{0 \mathrm{r}}\right), \\
& N_{0 \mathrm{r}}=\dot{L}_{0 \mathrm{r}}+\omega_{\mathrm{r}} \times L_{0 \mathrm{r}},
\end{aligned}
$$

where $F_{\mathrm{r}}, N_{0 \mathrm{r}}$ are all the forces and moments acting on the rider upper body. $m_{\mathrm{r}}$ is the mass of the rider upper body. $v_{0 \mathrm{r}}$ and the $\omega_{\mathrm{r}}$ are the velocity and the angular velocity of the rider upper body. $L_{0 \mathrm{r}}$ is the angular momentum of the rider upper body.

By combining the force and moment equations of the three bodies appropriately to cancel the internal forces and moments yields the following equations of motion for the entire system:

$$
\begin{aligned}
& F=F_{\mathrm{v}}+F^{\prime}+F_{\mathrm{r}}, \\
& N=N_{0 \mathrm{v}}+R_{\mathrm{v}} \times F_{\mathrm{v}}+N_{0}^{\prime}+R^{\prime} \times F^{\prime}+N_{0 \mathrm{r}}+R_{\mathrm{r}} \times F_{\mathrm{r}},
\end{aligned}
$$

where $F$ and $N$ are all the external forces acting on the rear assembly coordinate origin and all the external moments.

\subsubsection{External forces and moments}

The external forces and moments are presented as follows:

1. Side force

$$
F_{y}=Y_{1}+Y_{2} \text {. }
$$

The side forces are from the $y$-directional component of the front and rear tire forces.

2. Roll moment

$$
N_{x}=N_{x \mathrm{~g}}+M_{x \mathrm{f}}+M_{x \mathrm{r}} .
$$

The roll moments are from the gravity force and the $x$-directional component of the front and rear tire external moments.

3. Yaw moment

$$
N_{z}=N_{z 1}+N_{z 2} .
$$

The yaw moments are from the torques generated by the front and rear tire forces on the rear assembly coordinate origin about $z$-axis.

4. Front fork moment

$$
N_{z}^{\prime \prime}=N_{z 1}^{\prime \prime}+N_{z \mathrm{~g}}^{\prime \prime}-B \dot{\delta}+T .
$$

The front fork moments are from the rider control torque and the torques generated by the normal force, front tire force and the steering damp about the steering axis of the front fork assembly.

\subsubsection{Tire force}

The external forces and moments of the electrical motorcycle come from the gravity and the tire forces. The tire forces and the moments are important parts of 


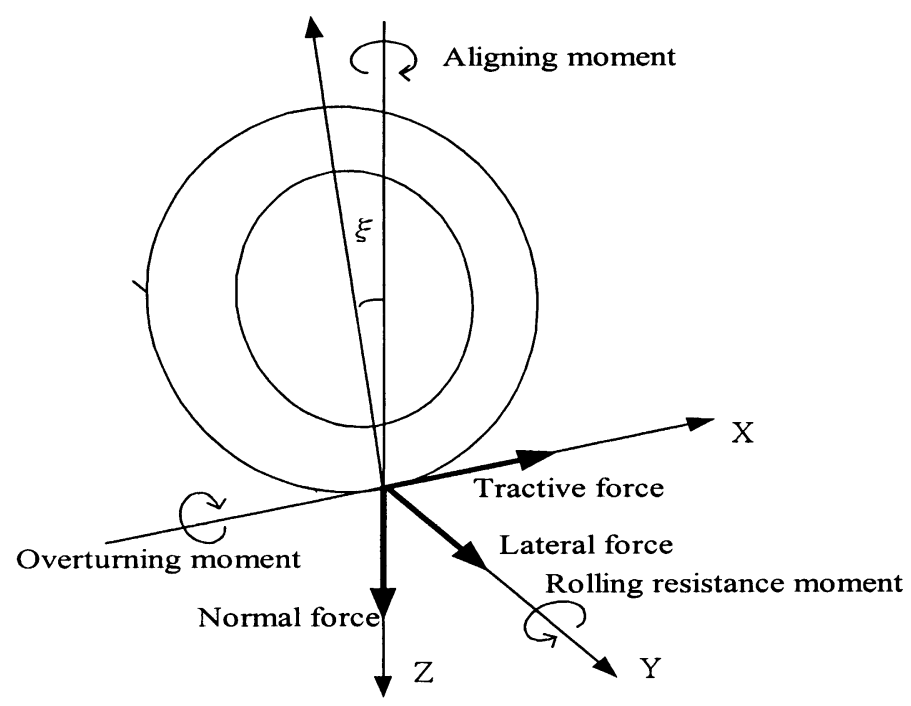

Fig. 4. Forces exerted on the tire.

constructing the equations of motion of the motorcycle analysis. A rolling tire is subjected to three components of forces and three components of torques in the vertical, longitudinal, and lateral directions as depicted in Fig. 4. The resulting forces and moments contain:

1. longitudinal force $F_{x}$, arising from longitudinal slip;

2. lateral force $F_{y}$ due to lateral slip, camber, and turn slip;

3. vertical force, denoted by $F_{z}$;

4. overturning moment $M_{x}$, generated by the lateral displacement of the contact patch when the wheel cambers;

5. rolling resistance moment $M_{y}$, resulting from the tire hysteresis properties;

6. aligning moment $M_{z}$, caused by asymmetrical side slip deformation, camber, turn slip and gyroscopic effects due to the rotating and deflected tire tread band, wheel rim, and tire side wall mass.

Referring to the research of Sharp [1], it is known that the aligning and rolling resistance moments do not affect obviously the dynamic behavior analysis. And because our research is on constant forward velocity motion, the longitudinal force can be ignored. The remaining forces and moment $F_{y}, F_{z}$ and $M_{x}$ are described below.

The linear model of an overturning moment can be obtained as:

$$
\begin{aligned}
& M_{x \mathrm{f}}=C_{\mathrm{mf}} \cdot \phi_{1}, \\
& M_{x \mathrm{r}}=C_{\mathrm{mr}} \cdot \phi_{2},
\end{aligned}
$$

where $C_{\mathrm{mf}}$ and $C_{\mathrm{mr}}$ are the overturning moment coefficients and $\phi_{1}$ and $\phi_{2}$ are the camber angles of the front and rear parts, respectively. 
The side force of tire: In case the sideslip and camber angles are small, the steadystate tire side forces are accurately described by linear functions of sideslip and camber angles of the form [1]:

$$
\begin{aligned}
& Y_{1}^{\prime}=C_{\alpha 1} \cdot \alpha_{1}+C_{\phi 1} \cdot \phi_{1}, \\
& Y_{2}^{\prime}=C_{\alpha 2} \cdot \alpha_{2}+C_{\phi 2} \cdot \phi_{2},
\end{aligned}
$$

where $\alpha$ is the slip angle, and $\phi$ is the camber angle.

Since the tire used in the electrical motorcycle is made of rubber, the response will be delayed for actual motion. The delayed response is decided by the relaxation length coefficient $\sigma$. For forward velocity $u$, the real tire side force and the steadystate tire side force have the following relationship:

$$
\begin{aligned}
& \frac{\sigma_{1}}{u} \cdot \dot{Y}_{1}+Y_{1}=Y_{1}^{\prime}, \\
& \frac{\sigma_{2}}{u} \cdot \dot{Y}_{2}+Y_{2}=Y_{2}^{\prime} .
\end{aligned}
$$

\subsubsection{The equations of motion}

Replacing the external forces and moments in the equations of motion of the entire system, adding the real tire equation of motion, and with the aid of the computer algebra software Mathematica, we can have the following six nonlinear equations of motion, where $u, v, w$ are the angular velocity components in $x$-, $y$-, $z$ direction, respectively; $p, q, r$ are the angular velocity components in $x$-, $y$-, $z$ direction, respectively:

\section{Side force equation}

$$
\begin{aligned}
m \dot{v}+ & m \cdot u \cdot r+m h \dot{p}+m^{\prime}\left(c_{1}\left(\dot{\delta}^{2}+\left(p^{2}-r^{2}\right)\right) \sin \delta+a^{\prime} \dot{r}\right) \\
- & 2 c_{1}(r \cos \lambda+p \sin \lambda) \sin \delta \dot{\delta}-c_{1} \dot{\delta}^{2} \sin \delta+m_{\mathrm{r}}\left(-\dot{r} a_{\mathrm{r}}+e \ddot{\phi}_{\mathrm{r}} \cos \phi_{\mathrm{r}}\right. \\
& \left.-e\left(r^{2}+\left(p+\dot{\phi}_{\mathrm{r}}\right)^{2}\right) \sin \phi_{\mathrm{r}}\right)=Y_{1}+Y_{2} .
\end{aligned}
$$

2. Yaw equation

$$
\begin{aligned}
I_{z x} \dot{p} & +I_{z z} \dot{r}+\left(I_{y y 2} \Omega_{2}+I_{y y 3} \Omega_{3}+I_{y y 1} \Omega_{1}\right) p+a^{\prime} m^{\prime} \dot{v}+a^{\prime} m^{\prime} u r \\
& +\left(I_{x z}^{\prime} \sin \lambda+I_{z z}^{\prime} \cos \lambda+m^{\prime} a^{\prime} c_{1} \cos \delta+c_{1}^{2} m^{\prime} \cos \lambda \sin ^{2} \delta\right) \ddot{\delta} \\
& +\left(I_{z x r}^{\prime} \cos \alpha+I_{z z r}^{\prime} \sin \alpha-a_{\mathrm{r}} e m_{\mathrm{r}}\right) \ddot{\phi}_{\mathrm{r}}-a_{\mathrm{r}} m_{\mathrm{r}} u r-a_{\mathrm{r}} m_{\mathrm{r}} \dot{v}+I_{y y 1} \Omega_{1} \sin \lambda \dot{\delta} \\
& -\dot{u}\left(c_{1} m^{\prime} \sin \delta+e m_{\mathrm{r}} \sin \phi_{\mathrm{r}}\right)+p r\left(c_{1} m^{\prime} h^{\prime} \sin \delta+m_{\mathrm{r}} e\left(h_{\mathrm{r}}+e \cos \phi_{\mathrm{r}}\right) \sin \phi_{\mathrm{r}}\right) \\
& +p^{2}\left(-c_{1} m^{\prime} a^{\prime}+a_{\mathrm{r}} e m_{\mathrm{r}} \sin \phi_{\mathrm{r}}\right)+p \dot{\delta}\left(2 c_{1} m^{\prime} a^{\prime} \sin \lambda \sin \delta\right) \\
& +\dot{\delta}^{2}\left(c_{1} m^{\prime} \sin \delta\left(f-\left(h_{1}-h_{1}^{\prime}\right)\right) \sin \lambda\right)+p \dot{\phi}_{\mathrm{r}}\left(2 a_{\mathrm{r}} e m_{\mathrm{r}} \sin \phi_{\mathrm{r}}\right) \\
& +r \dot{\phi}_{\mathrm{r}}\left(2 e^{2} m_{\mathrm{r}} \cos \phi_{\mathrm{r}} \sin \phi_{\mathrm{r}}\right)+\dot{\phi}_{\mathrm{r}}^{2}\left(a_{\mathrm{r}} e m_{\mathrm{r}} \sin \phi_{\mathrm{r}}\right)+r v\left(c_{1} m^{\prime} \sin \delta+e m_{\mathrm{r}} \sin \phi_{\mathrm{r}}\right) \\
& +r \dot{\delta}\left(2 c_{1} m^{\prime} \cos \lambda \sin \delta a^{\prime}+2 c_{1}^{2} m^{\prime} \cos \delta \sin \delta\right)=a \cdot Y_{1}-b \cdot Y_{2} .
\end{aligned}
$$


3. Roll equation

$$
\begin{aligned}
& I_{x x} \dot{p}+I_{z x} \dot{r}+\left(-I_{y y 2} \Omega_{2}-I_{y y 3} \Omega_{3}-I_{y y 1} \Omega_{1}+m h u\right) r+m h \dot{v} \\
& \quad+\left(I_{x x}^{\prime} \sin \lambda+I_{z x}^{\prime} \cos \lambda-c_{1} h^{\prime} m^{\prime} \cos \delta+c_{1}^{2} m^{\prime} \sin \lambda \sin ^{2} \delta\right) \ddot{\delta}+I_{x x r}^{\prime} \ddot{\phi}_{\mathrm{r}} \\
& \quad+\ddot{\phi}_{\mathrm{r}} e m_{\mathrm{r}}\left(h_{\mathrm{r}}\left(\cos \phi_{\mathrm{r}}-\sin \phi_{\mathrm{r}}\right)+e\right)-I_{y y 1} \Omega_{1} \cos \lambda \dot{\delta}-2 m^{\prime} h^{\prime} c_{1} r \cos \lambda \cdot \sin \delta \dot{\delta} \\
& \quad+2 p c_{1} m^{\prime} \sin \delta \dot{\delta}\left(c_{1} \cos \delta-h^{\prime} \sin \lambda\right)+p r\left(c_{1} a^{\prime} m^{\prime} \sin \delta-a_{\mathrm{r}} e m_{\mathrm{r}} \sin \phi_{\mathrm{r}}\right) \\
& \quad+p v\left(c_{1} m^{\prime} \sin \delta+e m_{\mathrm{r}} \cdot \sin \phi_{\mathrm{r}}\right)-\dot{\phi}_{\mathrm{r}}\left(2 e h_{\mathrm{r}} m_{\mathrm{r}} p \sin \phi_{\mathrm{r}}\right) \\
& \quad+c_{1} m^{\prime} \sin \delta \dot{\delta}\left(\left(h_{1}-h_{1}^{\prime}\right) \cos \lambda-h_{\mathrm{b}}\right)+r^{2}\left(c_{1} m^{\prime} h^{\prime} \sin \delta\right. \\
& \left.\quad-e m_{\mathrm{r}}\left(h_{\mathrm{r}}+e \cdot \cos \phi_{\mathrm{r}}\right) \sin \phi_{\mathrm{r}}\right) \\
& \quad=m h \cdot g \cdot \phi+m_{\mathrm{r}} \cdot g \cdot e \cdot \phi_{\mathrm{r}}-\left(k \cdot Z_{1}-m^{\prime} \cdot c_{1} \cdot g\right) \delta+C_{\mathrm{mf}} \phi_{1}+C_{\mathrm{mr}} \phi_{2} .
\end{aligned}
$$

4. Front fork hinge line equation

$$
\begin{gathered}
\left(I_{z x 0}^{\prime} \cos \lambda+I_{z z 0}^{\prime} \sin \lambda+c_{1} h^{\prime} m^{\prime}\right) \dot{p}+\left(-I_{z x 0}^{\prime} \sin \lambda+I_{z z 0}^{\prime} \cos \lambda+c_{1} a^{\prime} m^{\prime}\right) \dot{r} \\
+\left(I_{z z 0}^{\prime}+c_{1}^{2} m^{\prime}\right) \ddot{\delta}+c_{1} m^{\prime} \dot{v}+I_{y y 1} \Omega_{1} p \cos \lambda+\left(c_{1} m^{\prime} u-I_{y y 1} \Omega_{1} \sin \lambda\right) r \\
-c_{1}^{2} m^{\prime} \sin \left(\left(p^{2}+r^{2}\right)+2 \dot{\delta}(r \cos \lambda+p \sin \lambda)+\dot{\delta}^{2}\right) \\
=k \cdot Y_{1}+\left(k \cdot Z_{1}-m^{\prime} \cdot c_{1} \cdot g\right)(\phi+\delta \sin \lambda)+B \dot{\delta}-T .
\end{gathered}
$$

5. Front tire equation

$$
Y_{1}^{\prime}=C_{\alpha 1} \cdot \alpha_{1}+C_{\phi 1} \cdot \phi_{1} .
$$

6. Rear tire equation

$$
Y_{2}^{\prime}=C_{\alpha 2} \cdot \alpha_{2}+C_{\phi 2} \cdot \phi_{2} \text {. }
$$

\subsection{Linearization of the equations of motion}

With the aid of the computer algebra software Mathematica, the nonlinear equations of motion can be written compactly in the matrix form:

$$
\dot{x}=g(x, u), \quad \text { where } x=\left[\begin{array}{llllllll}
v & \dot{\phi} & r & \dot{\delta} & \phi & \delta & Y_{1} & Y_{2}
\end{array}\right]^{\mathrm{T}} \text {. }
$$

Because the nonlinear equations of motion are too complex for analysis, the nonlinear equations are expanded as a Taylor series in a specific point $\left(x_{0}, u_{0}\right)$ and $\phi=0$. Keeping only the first- and zero-order terms and neglecting higher-order terms, the nonlinear equations can be transferred to the following first-order linearized form:

$$
\begin{aligned}
\Delta \dot{x}_{i}= & \left.\sum_{j=1}^{n} \frac{\partial g_{i}(x, u)}{\partial x_{j}}\right|_{x_{0}, u_{0}} \Delta x_{j}+\left.\sum_{j=1}^{p} \frac{\partial g_{i}(x, u)}{\partial u_{j}}\right|_{x_{0}, u_{0}} \Delta u_{j} \\
& \left(x_{0}, u_{0}\right): \text { linearization point. }
\end{aligned}
$$

The above simultaneous linear differential equations can be written in a Laplace transformed matrix form:

$$
A \cdot X=B \cdot U,
$$


where $A$ and $B$ are the coefficient matrices of $s$, and the vector of states and the controls are $X$ and $U$ :

$$
\begin{aligned}
X & =\left[\begin{array}{llllll}
v & \phi & r & \delta & Y_{1} & Y_{2}
\end{array}\right]^{\mathrm{T}}, \\
U & =\left[\begin{array}{ll}
T & \phi_{\mathrm{r}}
\end{array}\right]^{\mathrm{T}} .
\end{aligned}
$$

The details of $A$ and $B$ matrices in (31) are listed in Appendix A.

\subsubsection{Linearization points of straight-line and cornering motions}

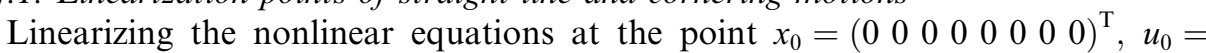
$\left(\begin{array}{ll}0 & 0\end{array}\right)^{\mathrm{T}}$, we obtain the equations of motion of straight-line motion, the same as those derived by Weir and Mears. On the other hand, the steady-state cornering equations [12] are employed to solve the instantaneous balance solution of $\left(x_{0}, u_{0}\right)$ to linearize the nonlinear equations to obtain cornering motion of motorcycles.

\subsection{Forward transfer function of motorcycle}

For hands free control, the steering torque $T$ is free considering only the rider body leaning motion. Substituting parameter values into (31), and manipulating algebraically, the system forward transfer function of roll angle to rider upper body lean at various forward velocities is derived as

$$
G(s ; u)=\frac{q(s ; u)}{p(s ; u)},
$$

where the numerator $p(s ; u)$ and the denominator $q(s ; u)$ are both of 8 degree polynomials. For example, when the motorcycle velocity is $30 \mathrm{~km} / \mathrm{h}$, the polynomials are:

$$
\begin{aligned}
p(s)= & -18.9543 s^{8}-1837.14 s^{7}-146023 s^{6}-7.69163 \times 10^{6} s^{5}-2.2893 \times 10^{8} s^{4} \\
& -5.21714 \times 10^{9} s^{3}-3.07984 \times 10^{10} s^{2}-7.42228 \times 10^{10} s+9.38629 \times 10^{10}, \\
q(s)= & 5.11302 s^{8}+496.491 s^{7}+35968.2 s^{6}+1.88069 \times 10^{6} s^{5}+5.19297 \times 10^{7} s^{4} \\
& +1.1513 \times 10^{9} s^{3}+7.48944 \times 10^{9} s^{2}-8.72078 \times 10^{9} s-6.61861 \times 10^{10}
\end{aligned}
$$

\subsection{Time delay of the rider control behavior}

It is assumed that there is a reaction for the rider that is conditioned by a firstorder dynamic system having a time constant, $\operatorname{Tr}[2,11]$. The time constant, $\operatorname{Tr}$, may be considered as a physiological rider constant, which may vary for different riders. To account for the effect of a regulation task for a rider on a motorcycle, $\mathrm{Tr}$ is set as $0.2 \mathrm{~s}$. Moreover, the Padé approximation is used to express the effect of time delay [10]. A time delay is approximated via the following formula:

$$
\exp \left(-t_{\mathrm{d}} s\right) \approx \frac{\left(1-0.5 t_{\mathrm{d}} s\right)}{\left(1+0.5 t_{\mathrm{d}} s\right)}
$$




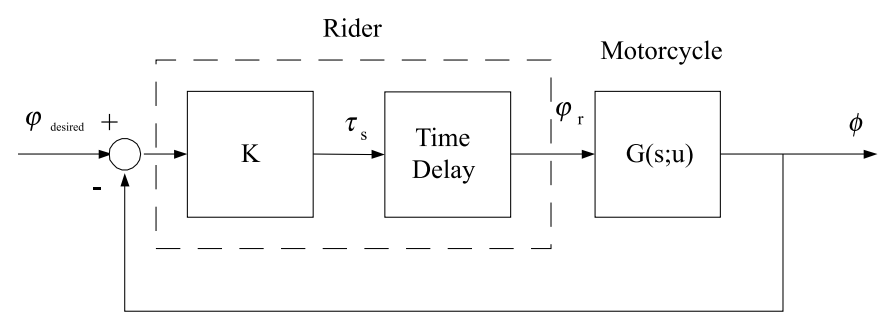

Fig. 5. The block diagram for proportional feedback control.

By using the Padé approximation, the effect of time delay representing rider response is approximated as

$$
\exp (-0.2 s) \approx \frac{(10-s)}{(10+s)}
$$

\subsection{Rider lean as a simple feedback control}

We can use the forward transfer function $G(s ; u)$ (roll angle to rider upper body lean angle) to determine whether or not a hands free rider can successfully stabilize the vehicle with only the upper body inclining left and right on the motorcycle seat [3]. The simplest possible control loop, a proportional control with a time delay, is placed around this transfer function as shown in the block diagram of Fig. 5. This type of control indicates that the rider is leaning his upper body in proportion to the amount that the motorcycle is leaning. The closed-loop transfer function becomes

$$
\frac{\phi}{\phi_{\text {desired }}}=\frac{k \exp (-0.2 s) G(s ; u)}{1+k \exp (-0.2 s) G(s ; u)} .
$$

\section{Straight line motion stability and perception of riding comfort}

Stability analysis of the eigenvalues of the characteristic equations of $G(s ; u)$ shows that there are three principal modes of motion for the lateral-directional dynamics [1,2]. They are:

Capsize mode: It means the roll motion of the motorcycle. This mode can be either stable or slightly unstable, leading to a gradual roll divergence in the absence of rider control.

Weave mode: It consists of a second-order motion involving combined roll and yaw of the total vehicle. It is usually well damped, but it can become oscillatory or even unstable depending on operating condition, vehicle configuration, rider control actions, etc.

Wobble mode: It consists of a second-order motion involving the response of the front fork assembly about its axis of rotation. Its natural frequency is relatively 
independent of velocity, but the damping significantly depends on velocity, rake and trail geometry, front fork assembly property, the front fork damper, etc.

These three modes can be viewed by the following root locus plot.

\subsection{Root locus}

The root locus plots for the motorcycle with (left plot) and without (right plot) control under various forward velocities are shown in Fig. 6. From the left plot of Fig. 6, which corresponds to $k=2.2$, it is seen that all roots lie on the left half of the $s$-plane under various forward velocities. It means a rider can use only leaning control to upright stabilize the motorcycle at various forward velocities. When the control gain $k=0$, i.e., the motorcycle is uncontrolled, all capsize mode roots lie on the right half of the $s$-plane, as shown in the right plot of Fig. 6 . The motorcycle is unstable, as is intuitively clear.

\subsection{Bode plot: gain margin and damping ratio}

In the control theory, the Bode plot of the open-loop transfer function $G(s ; u)$ is used to determine the stability and relative stability margins of closed-loop stable systems. Fig. 7 is the Bode plot of the open-loop transfer function $G(s ; u)$ for forward velocities varying from 25 to $60 \mathrm{~km} / \mathrm{h}$ every $5 \mathrm{~km} / \mathrm{h}$. It is found that for velocities between 35 and $55 \mathrm{~km} / \mathrm{h}$, the Bode plots are quite the same. Thus, for discussions in the following, the transfer function $G(s ; 35)$ is representative of $G(s ; u)$ for $u$ between 35 and $55 \mathrm{~km} / \mathrm{h}$.

\subsection{Stable region}

From the closed-loop transfer function of the proportional feedback control system in Fig. 5, we can determine the $k$-value of the stable region for various for-

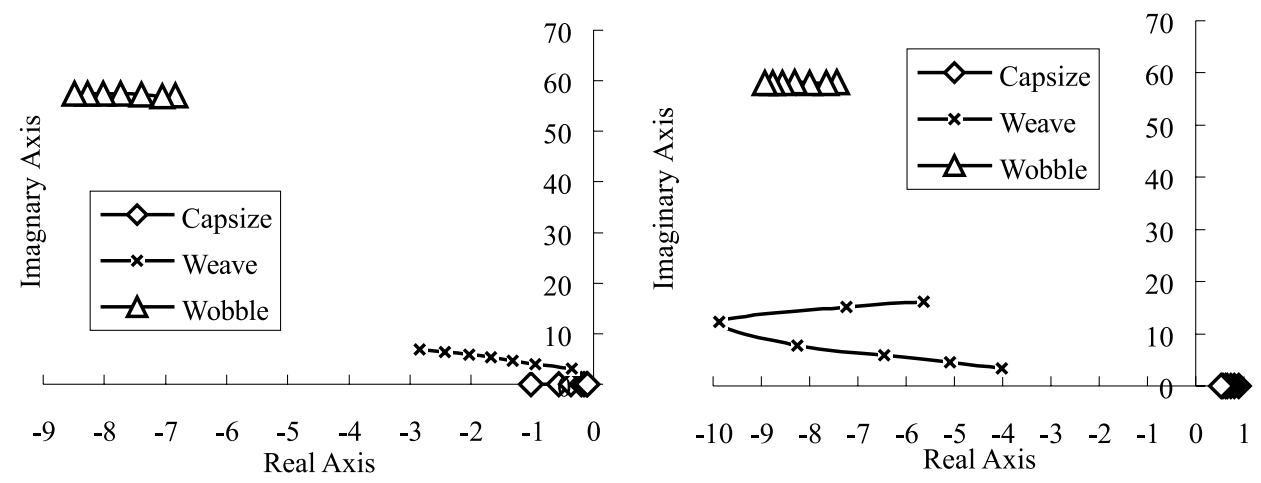

Fig. 6. The root locus plots for the motorcycle with (left) and without (right) control at various forward velocities. 

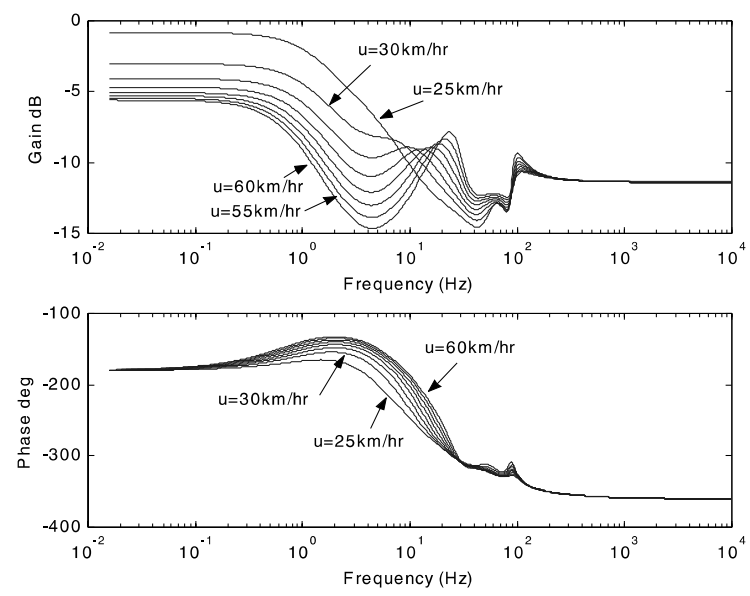

Fig. 7. The Bode plot for the open-loop transfer function $G(s ; u)$ for different velocities.

ward velocities via the Routh-Hurwitz criterion [10]. The stable regions of $k$ for various forward velocities are listed in Table 1, and plotted in Fig. 8. It is interesting that the stable region of $k$-value shown in Fig. 8 is increasing and becomes higher with the increase of forward velocities.

Calculating from the Bode plot of the open-loop transfer function $G(s ; u)$, we can find the closed-loop system gain margins for various forward velocities. The gain margin indicates that we can increase the gain by using the gain margin as a factor before the system becomes unstable.

In addition, we can determine the damping ratio $\zeta$ of the closed-loop system. From the definition of phase margin in the Nyquist plot, using simple geometry, we obtain

$$
\zeta \approx \sin \frac{\mathrm{PM}}{2},
$$

where PM is the phase margin of open-loop transfer function [10]. Because the transfer functions are different for various forward velocities and feedback gain values $k$, there is a maximum damping ratio for a gain value $k$ in each transfer

Table 1

The stable region of $k$ for various forward velocities

\begin{tabular}{llll}
\hline Forward velocity $(\mathrm{km} / \mathrm{h})$ & Stable region of $k$ & Damping ratio & Optimal $k$ \\
\hline 25 & $1.10<k<1.93$ & 0.130 & 1.39 \\
30 & $1.41<k<2.57$ & 0.222 & 2.04 \\
35 & $1.59<k<2.97$ & 0.273 & 2.55 \\
40 & $1.72<k<3.29$ & 0.310 & 2.75 \\
45 & $1.78<k<3.59$ & 0.333 & 2.96 \\
50 & $1.85<k<3.87$ & 0.347 & 3.04 \\
55 & $1.88<k<4.14$ & 0.358 & 3.08 \\
60 & $1.91<k<4.40$ & 0.367 & 3.14 \\
\hline
\end{tabular}




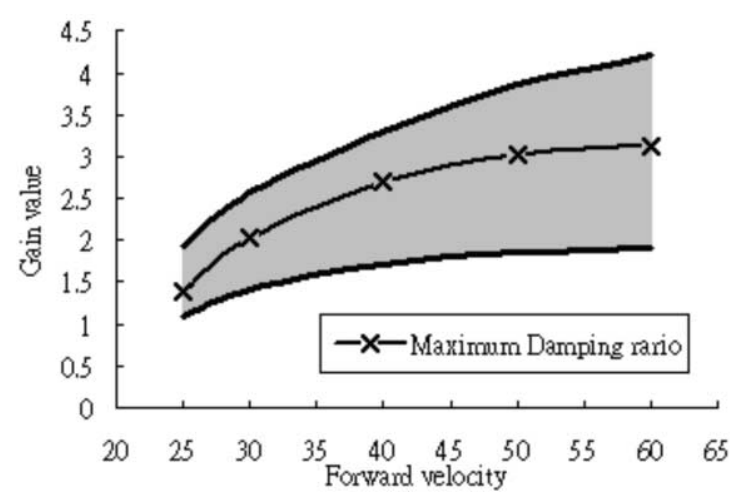

Fig. 8. The stable gain margin at various forward velocities.

function of different velocities. The maximum damping ratios along with $k$-value at various forward velocities are also listed in Table 1 and shown in Fig. 8.

The $k$-values corresponding to the maximum damping ratio at various forward velocities are also plotted in Fig. 8. It is clear that the maximum damping ratio value under various forward velocities is increasing as the velocity increased. This is again because the highest velocity of the electrical motorcycle is only at the moderate velocity in the conventional motorcycle model. So the damping ratio value will reach a better value with the velocity increasing.

\subsection{Step responses}

The effect of damping ratio can be viewed in a step function input response plot of the closed-loop system. For example, when the forward velocity is at $30 \mathrm{~km} / \mathrm{h}$, the maximum damping ratio value is 0.222 with the gain value $k=2.04$. It is found that when the damping ratio reaches a maximum value, the corresponding value $k$ is better for control. This can be seen from the step responses of the closed-loop transfer function. For comparisons, the responses for different gain values, $k=1.84,2.04$, 2.24, are plotted in the left one of Fig. 9, and the step responses for the maximum and the minimum stable gain values, $k=1.42,2.56$, are plotted in the right one of Fig. 9 .

When the feedback control gain value $k$ increases, e.g., $k=2.24$, it becomes an underdamped response, and there will be an overshoot happening. When the feedback control gain value $k$ decreases, e.g., $k=1.84$, it becomes the overdamped response, and the response becomes tardy. When the feedback gain value reaches the upper or lower stable margin, the step responses become almost unstable as shown in the right one of Fig. 9.

\subsection{Perception of riding comfort issue}

We wonder how the rider can have a better comfortable feeling under the stable feedback control gain. From discussions above, the comfort of rider control be- 

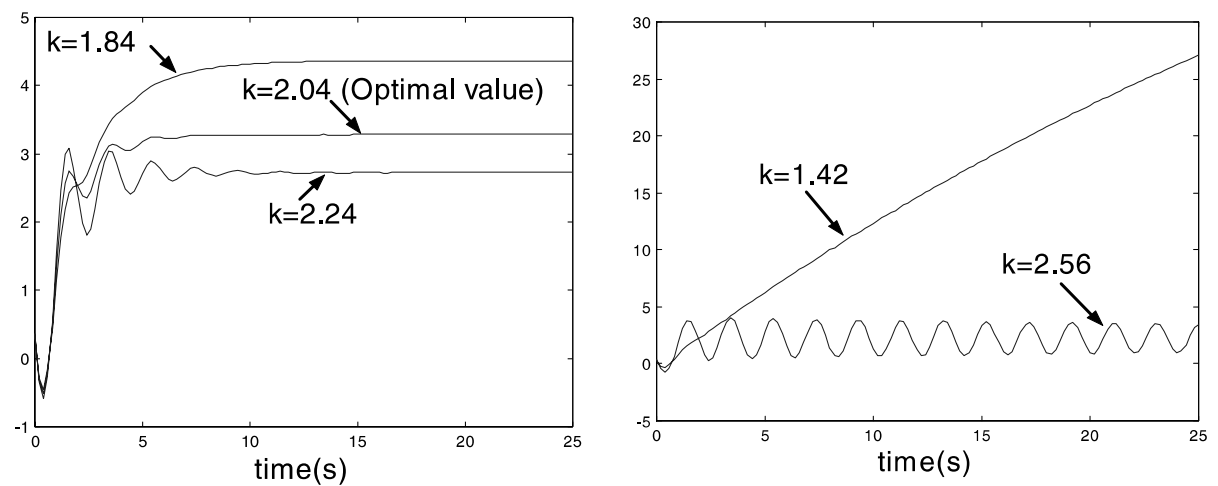

Fig. 9. The step responses for different gain values at forward velocity $u=30 \mathrm{~km} / \mathrm{h}$.

havior can be described by the damping ratio, which can be computed from the phase margin of the Bode plot of open-loop transfer function. We can say that when the damping ratio increases, the rider will have a feeling of better comfort, so that the damping ratio acts as a measure of perception of riding comfort for the design of the motorcycle parameters.

\subsection{Cornering motion}

The motion of a motorcycle is a combination of a series of linear and cornering motions. It is thus of importance to investigate the cornering motion [13-15]. The driving velocity of an electrical motorcycle is usually below $50 \mathrm{~km} / \mathrm{h}$. If the cornering radius is smaller than $10 \mathrm{~m}$, because of the limit of the camber angle of tire, the motorcycle forward velocity should be slow. At low speed cornering motion, the rider cannot use only the upper body leaning motion to maintain the stability of the motorcycle. Now we consider a motorcycle is turning under different cornering radii (specifically $5,10,20,40,60,80,100 \mathrm{~m}$ ), where larger radius corresponds to straight-line motion, and forward speeds (specifically $35,45,55 \mathrm{~km} / \mathrm{h}$ ). Using the same analysis method as straight-line motion, we get the stable region and damping ratio in different riding conditions of cornering motion summarized in Fig. 10. From the first three charts of Fig. 10, it is found that the stable margin value is lower as the cornering radius is smaller. This trend becomes more obvious at higher forward velocity. The lower stable margin is due to the centrifugal force effect during cornering motion. In other words, the rider can keep the motorcycle in a suitable leaning angle by a smaller upper body leaning motion. The damping ratios under different forward velocities and cornering radii are also computed in bar graph of Fig. 10, in which a small radius cornering is simulated to highlight the effect of cornering. It is found that the damping ratios are not sensitive to motion patterns, i.e., cornering or straight-line motions. 

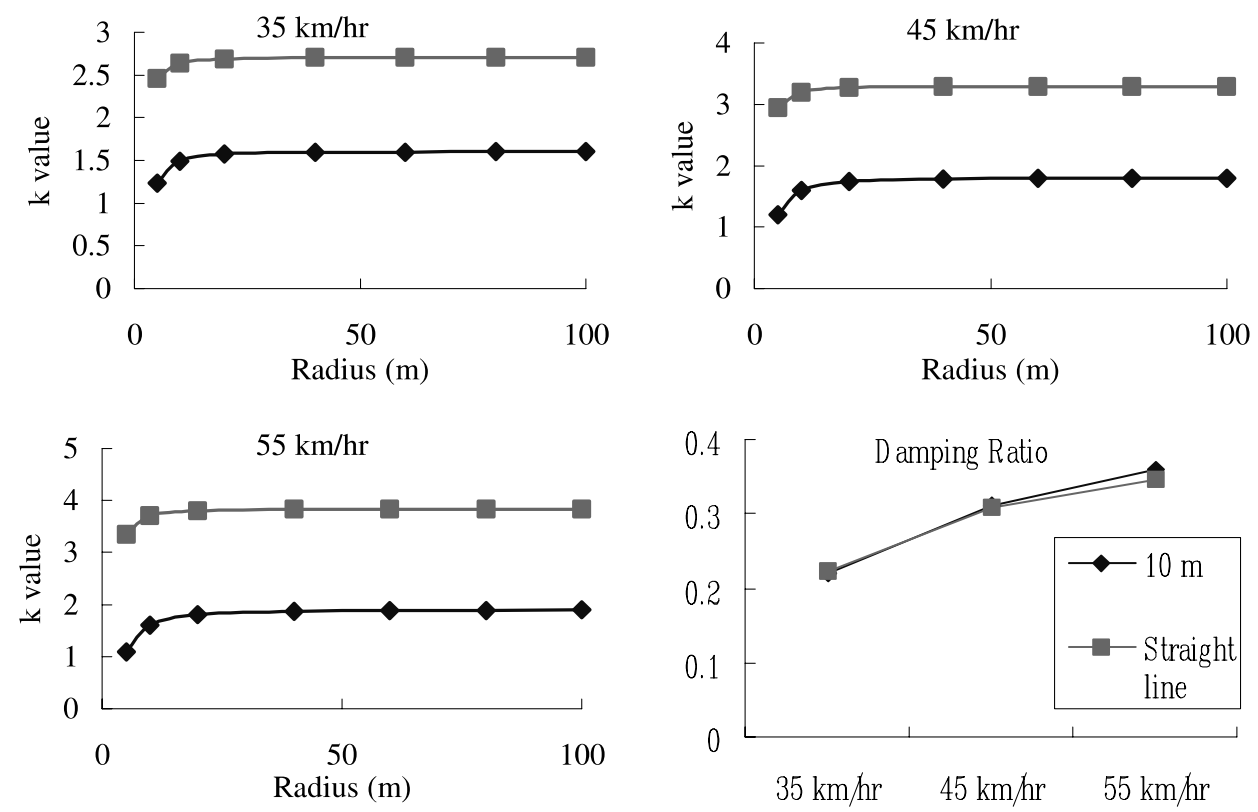

Fig. 10. The stable region and damping ratio during cornering motion.

\section{The effect of design variables on perception of riding comfort}

In the previous section, we derived dynamics of an electrical motorcycle controlled by rider upper body lean motion by Newton Mechanics. With the frequency domain analysis method of Bode plot in the control theory, the stable region and the perception of riding comfort of the rider-motorcycle system can be found by the gain margin and the phase margin of the system. The model data and parameter values used in the analyses of stability and perception of riding comfort are directly measured from the Jing-Shing-Fa electrical motorcycles manufactured in Taiwan. For the motorcycle design variables, Sharp [1] had discussed the effects of design variables changing on a single motorcycle (without rider control actions). However, the research in the perception of riding comfort issue of the rider-motorcycle system is lacking in general. In this section, we will discuss the effects of the main design variables of the electrical motorcycle on the driving stability and the perception of riding comfort of the rider-motorcycle system in some detail.

\subsection{The effect of weight reduction on the stability and perception of riding comfort}

From the component of the assembly of the electrical motorcycle, we can find that the weights of the front fork assembly and the rider upper body are lighter compared to the rear assembly, and the regions of the design variables are small. Therefore, the 

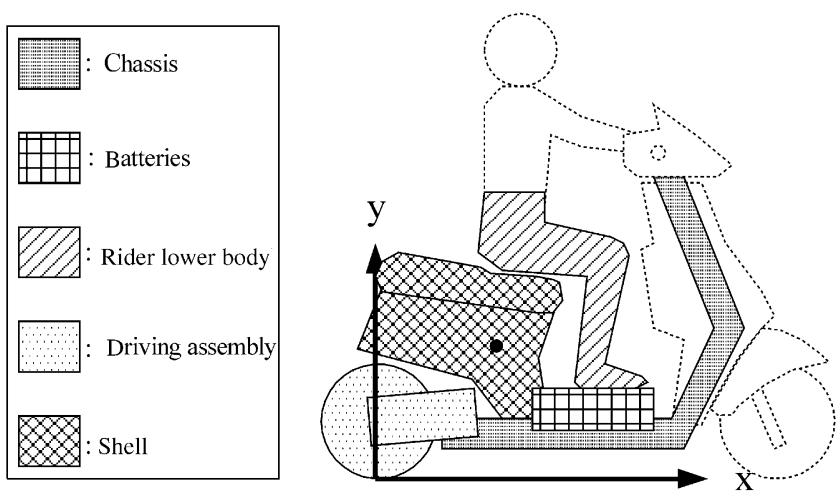

Fig. 11. The main parts of the electrical motorcycle.

main changeable part in weight and the mass distribution is the rear assembly of the motorcycle. We decompose the rear assembly into five main parts (Fig. 11):

1. The chassis

2. The batteries

3. The rider lower body

4. The motor, rear wheel and transmission parts

5. The shell of the motorcycle

The third-generation electrical motorcycle is made with the goal of lightening to save the battery power consumption. We expect to lighten the weight of electrical motorcycle from 120 to $90 \mathrm{~kg}$. It is undertaken from the three ways of the battery, chassis and transmission. Replacing the original lead-acid battery by the high energy density Li-on battery can reduce the weight by about $20 \mathrm{~kg}$. Using the aluminum alloy as the makings of the chassis can reduce the weight by about $7 \mathrm{~kg}$. In addition, using the one-stage transmission could reduce the weight of the transmission part by about $3 \mathrm{~kg}$. Although the lightening of the weight will not change the structure of the motorcycle, it will induce the change of the mass center position and the moment of inertia, and may affect the stability and perception of riding comfort of the electrical motorcycle under riding. To realize the effect of such a change, we analyze the effect of the changing of mass center position and the moment of inertial on the stability and perception of riding comfort under the same assembly distribution. By the discussion in Section 3.6, it is known that for the riding speed ranges of electrical motorcycle, the characteristics of cornering and straight-line motions are quite similar. Therefore, we use only the straight-line motion to analyze the effect, and the results are listed in Table 2.

From Table 2, when the weight is reduced from 120 to $90 \mathrm{~kg}$, the mass center position is moving backward and upward and the stable region is slightly shrunk, however, the damping ratio is almost the same. Comparing with a 50 cc gasolinepowered motorcycle, the weight of the third generation motorcycle is still heavier. 
Table 2

The effect of weight reduction on stability and damping ratio

\begin{tabular}{llll}
\hline $\begin{array}{l}\text { Total weight of } \\
\text { motorcycle }\end{array}$ & $\begin{array}{l}\text { Mass center position } \\
(x, y)\end{array}$ & Stable region & Damping ratio \\
\hline 120 & $(54.3,40.2)$ & $1.443<k<2.691$ & 0.293 \\
105 & $(53.0,41.6)$ & $1.569<k<2.698$ & 0.294 \\
90 & $(52.5,44.1)$ & $1.674<k<2.639$ & 0.287 \\
\hline
\end{tabular}

Hence, the goal of weight reduction of the third generation electrical motorcycles is a feasible design goal, since no obvious influence is observed on the stability and the perception of riding comfort.

\subsection{The effect of the weight distribution on the perception of riding comfort}

Another factor, which may affect the perception of riding comfort, is the position of the rear assembly mass center of the electrical motorcycle. The major element affects the rear assembly mass center position is the location where the heavy leadacid batteries are placed. Because all styles of the electrical motorcycle are designed as the scooter type, there is a limitation in the allowable position range of the rear assembly mass center affected by the distribution of the batteries. Measuring from a real electrical motorcycle made in Taiwan, the ranges of the mass center are $45.5-56.9 \mathrm{~cm}$ from the rear wheel axis in horizontal direction and $38-48.5 \mathrm{~cm}$ from the ground level in vertical direction. In this case study, the achievable range of batteries and the corresponding mass center is shown in Fig. 12.

Now we investigate the effect on the damping ratio of the control system if the center of mass position changes. Using the same analysis method, Fig. 13 shows the maximum damping ratio values for different mass center positions.

From Fig. 13, we can clearly find that there is a maximum peak value in the mass center distribution range, and the damping ratio becomes very small when the mass
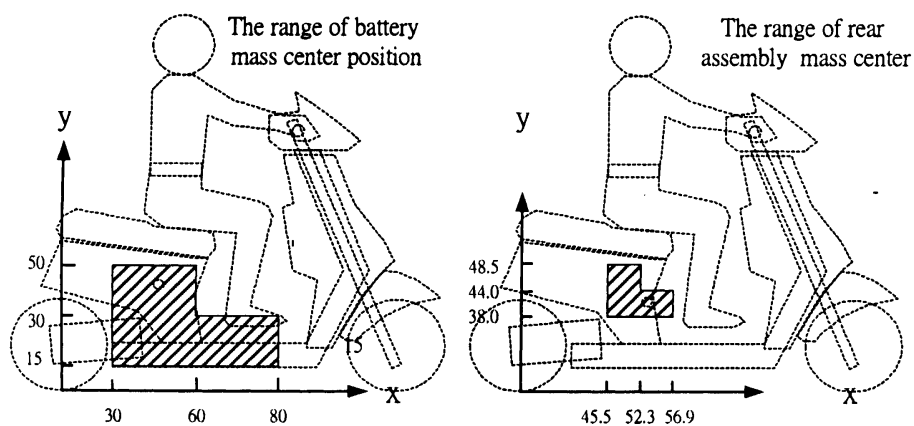

Fig. 12. Allowable locations (shaded region) of the batteries (left) and the corresponding rear assembly mass center moving range (right). 


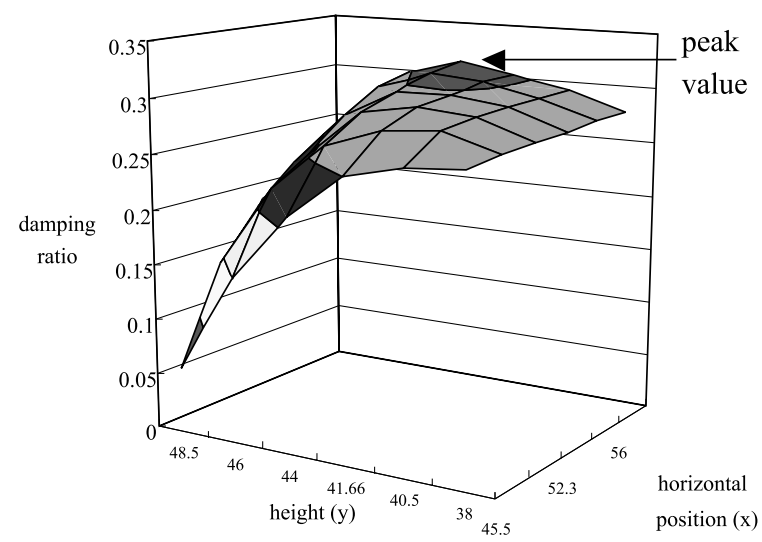

Fig. 13. The maximum damping ratio for different mass center positions.

center moves to the rear close to the rear wheel axis and the top of the range. It means that when the mass center is in the rear-top position, the rider is almost unable to stabilize the motorcycle by the upper body lean motion. Besides, it is also found that the more forward the mass center, the higher the maximum damping ratio is, and the maximum damping ratio appears in the front-middle position of the allowable range.

4.3. The effects of the wheelbase, the front fork rake angle and the trail distance on the stability and perception of riding comfort

Besides the weight change and distribution, there are three parameters, which will affect the stability and the perception of riding comfort. They are wheelbase, front fork rake and the trail distance of the electrical motorcycle.

\subsubsection{Wheelbase}

The wheelbase of the motorcycle is an important parameter. We increase and decrease the wheelbase by an amount of 10 and $20 \mathrm{~cm}$, separately. Analyzing the stability and perception of riding comfort of the motorcycle at $35 \mathrm{~km} / \mathrm{h}$ forward speed the results listed in Table 3 are obtained.

Table 3

The effect of wheelbase

\begin{tabular}{lll}
\hline Wheelbase & Stable region & Maximum damping ratio \\
\hline 100 & $1.18<k<2.46$ & 0.325 \\
110 & $1.40<k<2.59$ & 0.307 \\
120 (original design) & $1.60<k<2.71$ & 0.292 \\
130 & $1.79<k<2.81$ & 0.276 \\
140 & $1.97<k<2.91$ & 0.259 \\
\hline
\end{tabular}


Table 3 reveals that lengthening the wheelbase will reduce the stability region and the rider will use a larger motion to stabilize the motorcycle. In addition, lengthening the wheelbase will also reduce the damping ratio and the stability of capsize mode of the system so that the rider will feel less comfortable. On the contrary, from the paper of Sharp [1], we found that lengthening the wheelbase will decrease the damping of weave mode in low speed and increase the damping of weave mode in high speed. So in the high speed riding condition, a longer wheelbase is needed to improve the straight-line-running ability of motorcycles.

\subsubsection{Front fork rake angle}

The front fork rake angle can also affect the stability and the perception of riding comfort of the two-wheel motorcycle. The definition of the front fork rake angle is the angle between the plumb line and the front fork. By the same analysis method as above, the effect on stability and perception of riding comfort by changing the front fork rake angle from $15^{\circ}$ to $35^{\circ}$ is summarized in Table 4 .

The results of Table 4 indicate that for the perception of riding comfort, decreasing the front fork rake angle (steepen) will increase the stable region and the damping ratio. In other words, the rider will have a better perception of riding comfort. On the other hand, increasing the front fork rake angle will decrease the stable range and the damping ratio.

\subsubsection{Front wheel trail distance}

Another important parameter when considering the effect of the front fork rake angle is the front wheel trail distance. The definition of the front wheel trail distance is the distance between the tire-ground contact point and the front fork axis in the $x^{\prime}$ direction. Analyzing the effect of the front wheel trail distance on the stability and the perception of riding comfort in various front fork rake angles, we have the results listed in Table 5.

From Table 5, there should be a suitable trail distance for various front fork rake angles. An observation of the tendency is: as the front fork rake angle increases, the front wheel trail distance has to increase correspondingly to improve the stability and the perception of riding comfort. In addition, there is a maximum damping ratio for each trail distance under different rake angles. Besides, for the same trail distance,

Table 4

The effect of rake angle

\begin{tabular}{lll}
\hline Rake angle $\left(^{\circ}\right)$ & Stable region & Maximum damping ratio \\
\hline 15 & $1.71<k<3.10$ & 0.349 \\
20 & $1.64<k<2.90$ & 0.330 \\
25 (original design) & $1.60<k<2.71$ & 0.292 \\
30 & $1.57<k<2.54$ & 0.244 \\
35 & $1.55<k<2.39$ & 0.200 \\
\hline
\end{tabular}


Table 5

The effect of front wheel trail distance

\begin{tabular}{|c|c|c|c|c|}
\hline \multirow[t]{2}{*}{ Trail distance } & & \multicolumn{3}{|l|}{ Rake angle } \\
\hline & & $15^{\circ}$ & $25^{\circ}$ & $35^{\circ}$ \\
\hline \multirow[t]{2}{*}{$0.102 \mathrm{ft}$} & Stable region & $1.22<k<2.25$ & $1.36<k<2.33$ & $1.55<k<2.39$ \\
\hline & $\begin{array}{l}\text { Max. damping } \\
\text { ratio }\end{array}$ & 0.245 & 0.230 & 0.200 \\
\hline \multirow[t]{2}{*}{$0.68 \mathrm{ft}$} & Stable region & $1.33<k<2.60$ & $1.60<k<2.71$ & $1.95<k<2.81$ \\
\hline & $\begin{array}{l}\text { Max. damping } \\
\text { ratio }\end{array}$ & 0.329 & 0.292 & 0.244 \\
\hline \multirow[t]{2}{*}{$0.34 \mathrm{ft}$} & Stable region & $1.71<k<3.10$ & $2.28<k<3.25$ & $2.98<k<3.41$ \\
\hline & $\begin{array}{l}\text { Max. damping } \\
\text { ratio }\end{array}$ & 0.360 & 0.250 & 0.054 \\
\hline
\end{tabular}

decrease of the rake angle reduces both the stable region and the perception of riding comfort.

\subsection{Discussions}

From the discussion above, weight reduction of the electrical motorcycle does not affect the stability and the perception of riding comfort. Besides, moving the location of batteries forward and upward to a suitable position, shortening the wheelbase, decreasing the front fork rake angle and a suitable trail angle is of use in achieving a better perception of riding comfort. This result is quite different from the traditional design impressions. As a convention of design, moving the batteries lower, lengthening the wheelbase and increasing the front fork rake angle will help to improve the stability of the motorcycle [1]. The difference first comes from the electrical motorcycle discussed in this paper belongs to a low speed range, so only the response of the low speed motion needs to be investigated. Second, our stability and perception of riding comfort analyses are based on a rider upper lean controlled motorcycle-rider system. The perception of riding comfort is determined by the maximum damping ratio of the system in the stable range. Because the rider employs only left-right lean motion to stabilize the motorcycle, so the more sensitive the motorcycle is, the quicker it responds to perturbation. In other words, the rider will have better perception of riding comfort. In this sense, the perception of riding comfort we discussed actually reflects the sensitivity of the stable motorcycle response.

The major difference with Sharp's paper [1] is that: Sharp discussed the stability of a straight-line-running single motorcycle without rider (and rider control). The discussions of design parameters focused on their effects on the weave mode (straight-line-running ability). Our discussions focus on the effect of design parameters on the perception of riding comfort under only rider upper body lean control (without steering control), and the major factor that affects the perception of riding 
comfort is the lateral directional motion sensitivity of the motorcycle under stable running.

\section{Conclusion}

In this study, a simple proportional feedback control is used to model the rider's delayed lean control for investigating a hands-free rider to drive an electrical motorcycle at moderate forward speeds. A frequency domain analysis of stability and perception of comfort of rider-motorcycle system is performed by using the Bode plots. From the gain and phase margins obtained from the Bode plots, the effects of rider leaning behavior on the relative stability and rider comfort for straight-line and cornering motions are studied quantitatively. From this study, the following results are obtained for electrical motorcycles controlled only by means of leaning of the rider body:

1. The gain margin and the phase margin obtained from the Bode plots can be regarded as a measure of the stability and perception of riding comfort of the rider-motorcycle system under rider upper body leaning motion. In contrast to [8], where steer is for stabilizing and lean for comfort purposes, the rider can maintain upright stability of a constant speed straight or cornering running electrical motorcycle and adjust perception of riding comfort by using only rider's upper body leaning action for a wide range of mediate speeds.

2. Under the rider upper body leaning control with time delay, the stable margin and maximum damping ratio are both increasing and become higher as the forward speed increases. The rider can have a better perception of riding comfort at a higher velocity.

3. The motorcycle driving actions on the city road, such as lane changing and overtaking another vehicle, all belong to the larger radius cornering motion. In the interest of perception riding comforts by using rider leaning, results of straight-line motion can stand for the riding conditions on the city road for the electrical motorcycle.

4. The change of design parameters such as mass center position, wheelbase, rake angle and trail distance indeed affects the perception of riding comfort of the rider. By the analyses, the designer of the electrical motorcycle can adjust the design parameters to achieve a better perception of riding comfort in the design process.

5. From the study of perception of riding comfort, we can find that the stable design should compromise with perception of riding comfort. For better perception of riding comfort, we should find out the maximum damping ratio of the ridermotorcycle system in the stable region.

These analysis results can be a useful reference for the design and performance evaluation of electrical motorcycles. The design considerations of parameters of 
electrical motorcycles should include not only the stability of the rider-motorcycle system at various speeds, but also have to include the comfort influence of the rider leaning behavior.

\section{Acknowledgements}

The authors thank the reviewers for very detailed and useful comments. This study was supported by National Science Council of ROC under contract AG20102. The authors also thank the valuable consulting from Prof. C.H. Wu of Northwestern University.

\section{Appendix A}

\section{A.1. Notations}

\section{Geometric parameters (Fig. 1)}

$a \quad$ distance from rear assembly mass center to front tire axle (along $x$ )

$a^{\prime} \quad$ distance from rear assembly mass center to front fork mass center (along $x$ )

$a_{\mathrm{r}} \quad$ distance from rider upper body mass center to rear mass center (along $y r$ )

$b \quad$ distance from rear assembly mass center to rear tire axle (along $x$ )

$c \quad$ distance from front fork hinge lint to front tire axle (along $x^{\prime}$ )

$c_{1} \quad$ distance from front fork hinge lint to front fork mass center (along $x^{\prime}$ )

$d_{1} \quad$ wheel diameter

$e \quad$ distance from torsion hinge lint to upper body mass center

$f \quad$ distance from rear assembly mass center to front fork hinge point (along $x)$

$h_{1} \quad$ distance from front fork hinge point to front axle (along $y^{\prime}$ )

$h \quad$ height of vehicle mass center (along $y$ )

$h^{\prime} \quad$ height of front fork assembly mass center (along $y$ )

$h_{\mathrm{b}} \quad$ height of front fork hinge point (along $y$ )

$h_{\mathrm{v}} \quad$ height of rear assembly mass center (along $y$ )

$\alpha_{\mathrm{r}} \quad$ torso hinge line incline angle (about the ground level)

$\beta \quad$ ground level incline angle (about to the earth surface)

$\lambda$ front fork hinge line inclination (frame rake angle)

$k \quad$ front wheel trail (along $x^{\prime}$, normally negative)

Mass and moment of inertia parameters (Fig. 1)

$\mathrm{cm}$ mass center of the total motorcycle assembly

$\mathrm{Cm}_{\text {rear }}$ mass center of the rear assembly

$\mathrm{cm}_{\mathrm{f}} \quad$ mass center of the front fork assembly

$c m_{\text {rider }}$ mass center of the rider upper body 
$m_{\mathrm{v}} \quad$ mass of the rear assembly including the low body of the rider

$m_{\mathrm{r}} \quad$ mass of the rider upper body

$m^{\prime} \quad$ mass of the front fork assembly

$\left[I_{x x}, I_{y y}, I_{z z}, I_{x z}\right]$ moment of inertia of the inertial frame

$\left[I_{x x 0}^{\prime}, I_{y y 0}^{\prime}, I_{z z 0}^{\prime}, I_{x z 0}^{\prime}\right]$ moment of inertia of the front fork frame

$\left[I_{x x r 0}, I_{y y r 0}, I_{z z r 0}, I_{x z r 0}\right]$ moment of inertia of the rider upper body frame

$\left[I_{y y 1}, I_{y y 2}, I_{y y 3}\right]$ moment of inertia of the front wheel, rear wheel and the engine rotor

Position, velocity, and force

$B \quad$ front fork damping

$\phi \quad$ motorcycle roll angle

$\phi_{\mathrm{r}} \quad$ rider upper body roll angle

$\delta \quad$ front fork steer angle

$\Omega_{1}, \Omega_{2}, \Omega_{3}$ angular velocity of front wheel, rear wheel and motor rotor

$p, q, r \quad$ angular velocity of the rear assembly body axis

$Z_{1} \quad$ vertical load at front tire contact patch

\section{A.2. Parameter values}

The values of main parameters used in simulations are listed as follows:

\begin{tabular}{|c|c|c|}
\hline Parameter & Value & Description \\
\hline$M$ & $170 \mathrm{~kg}$ & The total mass of the rider-motorcycle system \\
\hline$m_{\mathrm{v}}$ & $137 \mathrm{~kg}$ & $\begin{array}{l}\text { The mass of the rear part of the motorcycle } \\
\text { (including the rider's hip and legs) }\end{array}$ \\
\hline$m_{\mathrm{r}}$ & $20 \mathrm{~kg}$ & $\begin{array}{l}\text { The mass of the rider's upper body including } \\
\text { hands and head }\end{array}$ \\
\hline$h_{\mathrm{v}}$ & $0.38 \mathrm{~m}$ & The height of the mass center of the rear part \\
\hline$I_{x x o f}$ & $0.636 \mathrm{~kg} \mathrm{~m}^{2}$ & The moment of inertia of the front fork part \\
\hline$I_{z z o f}$ & $0.228 \mathrm{~kg} \mathrm{~m}^{2}$ & \\
\hline$I_{x x}$ & $65 \mathrm{~kg} \mathrm{~m}^{2}$ & $\begin{array}{l}\text { The moment of inertia of the rider-motorcycle } \\
\text { system }\end{array}$ \\
\hline$I_{x z}$ & $23 \mathrm{~kg} \mathrm{~m}^{2}$ & \\
\hline$I_{z z}$ & $4.9 \mathrm{~kg} \mathrm{~m}^{2}$ & \\
\hline$I_{x x o r}$ & $1.547 \mathrm{~kg} \mathrm{~m}^{2}$ & The moment of inertia of the rider upper body \\
\hline$a$ & $0.67 \mathrm{~m}$ & $\begin{array}{l}\text { The distance from the front and rear tire contact } \\
\text { point to the mass center along } x \text {-axis }\end{array}$ \\
\hline$b$ & $0.53 \mathrm{~m}$ & \\
\hline
\end{tabular}

\section{A.3. Matrices $A$ and $B$}

The matrices $A$ and $B$ are shown as follows: 


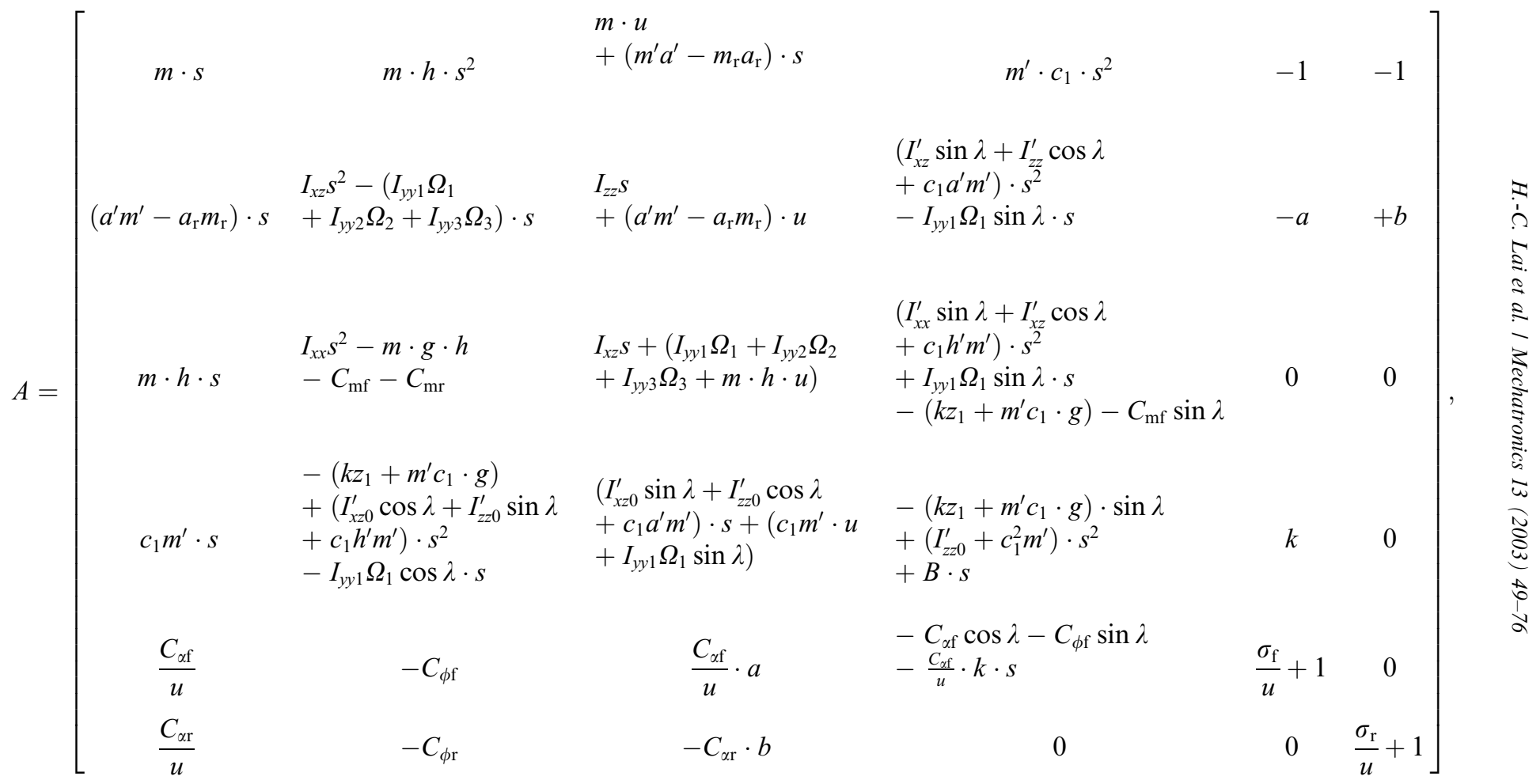




$$
B=\left[\begin{array}{cc}
0 & -m_{\mathrm{r}} e \cdot s \\
0 & -\left(I_{x z 0 \mathrm{r}}-a_{\mathrm{r}} m_{\mathrm{r}} e\right) \cdot s^{2} \\
0 & \left(I_{x x 0 \mathrm{r}}+m_{\mathrm{r}}\left(h_{\mathrm{r}}+e\right) \cdot e\right) \cdot s^{2}+m_{\mathrm{r}} \cdot g \cdot e \\
1 & 0 \\
0 & 0 \\
0 & 0
\end{array}\right]
$$

\section{References}

[1] Sharp RS. The stability and control of motorcycle. J Mech Eng Sci 1971;13(5):316-29.

[2] Weir DH, Zellner JW. Lateral-directional motorcycle dynamics and rider control. SAE Paper 780304, 1978.

[3] Craig MB. Open loop aspects of two wheeled vehicle stability characteristics. Ph.D. Thesis, University of Illinois at Urbana-Champaign, 1989.

[4] Wong JY. Theory of ground vehicles. New York: Wiley International; 1993.

[5] Genta G. Motor vehicle dynamics - modeling and simulation. Singapore: World Scientific; 1997.

[6] Yeh C, Edge YL. Handling analysis of a motorcycle with added cambering of the front frame. Vehicle Syst Dyn 1990;19:49-70.

[7] George L, Mosher LS. Motorcycle mechanics. Englewood Cliffs, NJ: Prentice-Hall; 1977.

[8] Katayama T, Aoki A, Nishimi T. Control behavior of motorcycle riders. Vehicle Syst Dyn 1988;17:211-29.

[9] Yokomori M, Oya T, Katayama A. Rider control behavior to maintain stable upright position at low speed. JSAE Rev 2000;21:61-5.

[10] Kuo BC. Automatic control system. 6th ed. Englewood Cliffs, NJ: Prentice-Hall; 1991.

[11] Liu TS, Chen JS. Nonlinear analysis of stability for motorcycle-rider systems. Int J Vehicle Des 1992;13:276-94.

[12] Krauter AI. Steady-state cornering of two-wheeled vehicles. J Appl Mech 1973:819-21.

[13] Cossalter V, Doria A, Lot R. Steady turning of two wheel vehicles. Vehicle Syst Dyn 1999;31:157-81.

[14] Riedl K, Lugner P. Naehere untersuchungen zur stationaren kurvenfahrt von einspurfahrzeugen. Vehicle Syst Dyn 1982;11:175-93.

[15] Ishii H, Tezuka Y. Considerations of turning performance for motorcycles. JSAE SAE Paper no. $972127,1997$. 